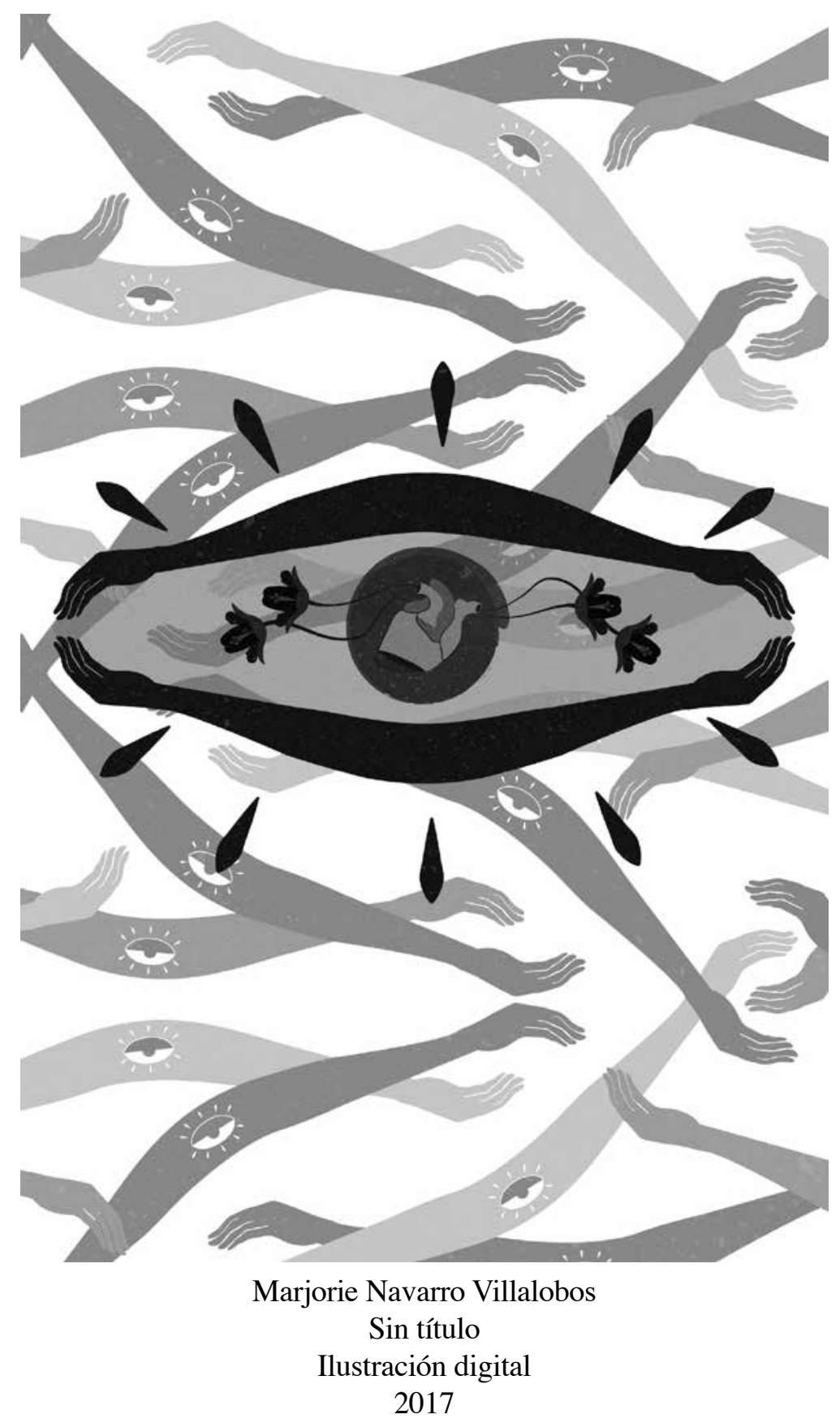




\section{Retrato de la homosexualidad y la homofobia en la literatura costarricense}

Joselyn Miranda González

Estudiante de Biología. Universidad Nacional

$\mathbf{L}$

a literatura, como otras artes, es una expresión de la humanidad, de su día a día, de sus miedos e ilusiones, de su historia pasada, presente y futura. Ella permite vestir los zapatos de otros y ver de cerca diferentes vidas y puntos de vista. En el presente ensayo se muestra un análisis/opinión sobre extractos de literatura erótica, en especial homosexual, escritos por autores costarricenses y recopilados en la antología La gruta y el arcoíris: antología de narrativa gay/lésbica costarricense de Alexánder Obando, quienes han expuesto en sus textos, personajes dentro de una sociedad que en la actualidad sigue con una mentalidad un tanto cerrada, conservadora y muchas veces mojigata e hipócrita.

Talvez se recuerde, en las noticias internacionales de 2016, titulares acerca de una gran masacre de personas homosexuales en un club nocturno de Orlando, Florida, la cual ocurrió a mediados de junio. La tragedia hizo eco en medios de comunicación masiva por varios días e incluso semanas, $\mathrm{y}$ ahora en la memoria de la comunidad
LGBT $^{1}$ pesan esas vidas violentamente interrumpidas; sin embargo, esto no solo debería lastimar a esa comunidad, debería lastimar a todos los seres humanos, los cuales, al parecer, han desplazado su humanidad por intolerancia, odio y discriminación. Y no hay que ir tan lejos, lamentablemente, para ejemplificar este triste panorama, porque en la pequeña, adorable y pura vida Costa Rica, a pesar de que ya no se practican las redadas policiales a bares de gays y lesbianas como se hizo en las décadas de los sesentas hasta los noventas $^{2}$, estas personas siguen sufriendo el rechazo y maltrato por una sociedad en extremo tradicionalista e influida de manera muy fuerte por la religión. Muchos de ellos y ellas siguen con un miedo latente de ser mirados con reprobación, incluso por sus familias. y ¿Acaso una madre o un padre pueden dejar de querer a un hijo o una hija por tener « $\langle\mathrm{x} » 0$ o $\langle\mathrm{y}\rangle$ orientación

1 Serrano, J. F. (2006: 103). Término para referirse a las personas Lesbianas, Gays, Bisexuales y Transgénero.

2 Obando, A. (2008). En el prólogo de «La gruta y el arcoíris» da un esbozo histórico de la gente LGBT en Costa Rica. 
sexual? Si todos los seres humanos están hechos de la misma materia orgánica-, tienen brazos, pies, ojos, boca y órganos vitales- entonces, ¿por qué tienen que esconderse en bares para homosexuales para evitar las malas caras llenas de prejuicios por sostener la mano del individuo que quieren o darse un beso? ${ }^{3}$

La situación del grupo LGBT se puede ver plasmada en muchos ámbitos (derechos civiles, sociedad, familia, ambiente laboral). No obstante, un área se ha encargado por mucho tiempo de evidenciar y mostrar la realidad que vive este grupo; ha combinado elementos ficticios basados en la cotidianeidad; el arte de la literatura ha construido historias que ejemplifican el vivir de individuos que intentan llevar una vida tan normal como la de cualquier otro, pero que ya sea dentro de la trama o fuera de esta, se enfrentan al ataque de la homofobia. Porque sí, hasta los mismos escritores, bien sean o no homosexuales, han sido víctimas de críticas en torno a su orientación sexual y no a la calidad literaria que puede presentar la obra como pieza de arte ${ }^{4}$. Estos poemas, novelas y cuentos han causado controversia tanto por la temática que manejan como por el estilo de escritura, unos más explícitos que otros, pero en su mayoría tan bien elaborados que muestran con elegancia y sensualidad la sexualidad humana, sin importar si es heterosexual u homosexual.

Antes de hablar a fondo sobre algunas de estas obras escritas por costarricenses, hay que tener claros conceptos como sexo,

3 Ulloa, D. (2016). Artículo de opinión publicado en el periódico La Nación, Costa Rica.

4 Obando, A. (2008). Ídem. orientación sexual y sexualidad; todos relacionados, pero no sinónimos uno del otro. El sexo se refiere a las características físicas y morfológicas que diferencian al humano entre hombre y mujer, es decir, sus órganos sexuales, por ejemplo, los externos: pene y vagina ${ }^{5}$. La orientación sexual es la atracción afectiva, emocional, romántica $\mathrm{y} / \mathrm{o}$ sexual que siente una persona hacia otra. Científicos han intentado por años definir el origen de la orientación sexual, pero muchos en la actualidad la consideran como un producto de la compleja combinación de elementos genéticos, cognitivos, biológicos, ambientales y hormonales; en la mayoría de las personas se define desde temprana edad o en la adolescencia ${ }^{6}$. Ser de una orientación sexual u otra es más que una simple escogencia, pero por siglos al homosexualismo se le trató como un desorden psiquiátrico o enfermedad tratada con terapia para «convertir a la persona en heterosexual». En cuanto al término sexualidad, es la expresión en pensamientos, creencias, actitudes, prácticas y relaciones basadas en el sexo de la persona, el género, la identidad sexual, la orientación sexual, la afectividad, el erotismo y la reproducción; y para cada individuo el modo de experimentarla es distinto ${ }^{7}$.

Carmen Naranjo, en su cuento «Trece partes de un amaneramiento», presenta a Adolfo, un hombre homosexual que desde temprana edad sintió afinidad por la ropa femenina y cuando alcanzó la edad para escoger una carrera, tuvo que pensar con

5 Arango de Montis, I. (2008: 4,5). Sexualidad Humana.

6 Maroto, A. (2006: 301,302). Homosexualidad y Trabajo Social.

7 Arango de Montis, I. (2008:4,5). Ídem. 
cuidado en algo donde pudiera tener la libertad suficiente para sentirse libre de cuchicheos malintencionados de la sociedad que lo hacía sentir como un degenerado; sin embargo, cuando finalmente escogió estudiar diseño de modas en Roma y lo anunció a sus padres, su madre se mostró colaboradora con él al ofrecerle sus ahorros, pero su padre enfureció por la noticia y lo llamó «maricón». Esto se puede ver en el siguiente párrafo:

[...] pensó en la biología, pues le gustaban los animales, las flores y los injertos. Además prefería andar libre por los campos que recorrer las calles hacinadas de gente vulgar y grosera. Pero los estudios exigían prácticas en campamentos con otros compañeros, lo que le daba terror porque tendrían que aglutinarse por miedo a estar cerca de él, seguros de que las desviaciones se contagian y para qué pasar un mal rato ${ }^{8}$.

[...] Habló con su madre, le declaró que quería ser modisto, aprender diseño de estilos, corte de ropa femenina y alta costura. [...] La madre le propuso darle los ahorros, pero el padre se enojó mucho, dijo que ya lo habían educado como si fuera una mujer y ahora le iban a facilitar un oficio de maricón?".

La situación de Adolfo es la de muchas personas LGBT, quienes incluso nunca llegan a «salir del clóset» frente a su familia por temor al rechazo por parte de seres tan queridos, así que optan por esconder su orientación sexual y, como si eso no fuera suficiente, condicionan sus futuros

8 Obando, A. (2008: 12). Autora original Carmen Naranjo.

9 Obando, A. (2008: 12). Ídem. profesionales por miedo al qué dirán por parte de la sociedad.

En la segunda parte del capítulo tres de la novela Mariposas negras para un asesino, Jorge Méndez Limbrick, relata con un leguaje entre lo elegante y familiar una carta/correo electrónico que Jackie le envía a su amiga Guillermina, donde le cuenta una experiencia íntima y a la vez cotidiana cuando existe atracción, amor y deseo entre dos personas:

[...] Nos besamos, fue hermoso, delicado, tierno $^{10}$.

[...] Me imaginé que íbamos a rodar por la alfombra como en esas películas baratas de Hollywood, o nos arrancaríamos la ropa como en una escena supererótica de "Bajos instintos". Fue todo lo contrario, mi amiga fue imponiendo pautas a nuestro juego en una complicidad tácita ${ }^{11}$.

[...] Existen muchas maneras de hacer el amor. La mayoría de las personas no lo podrían entender jamás. Creen que hacer el amor es la simple penetración. ¡Jamás! Esto es la gente vulgar. La gente que conoce el "ars amandi" sabe que son muchas las formas de lo erótico ${ }^{12}$.

No solo existen muchas maneras de hacer el amor, también hay muchos tipos de amor, pero en la cultura patriarcal y todavía machista del costarricense, se podrán topar en la calle gente que dice que la unión solo «debe y está bien» entre un hombre y una mujer. ¿Por qué? ¿Qué tiene de erróneo

10 Obando, A. (2008: 95,96). Autor original Jorge Méndez Limbrick.

11 Obando, A. (2008: 95,96). Ídem.

12 Obando, A. (2008: 95,96). Ídem. 
que dos personas sin importar su sexo se amen? Ser gays, lesbianas, bisexuales o transgénero solo les debería importar a la persona con la que se comparte la cama y no al mundo entero; con sus sentimientos $\mathrm{y}$ afectividad no dañan a nadie $\mathrm{y}$, no obstante, muchos se ofenden y oponen a estas uniones. Parece ser tanta la ofensa que inclusive el único matrimonio de lesbianas en Costa Rica, que fue posible gracias a un error del Registro Civil al tener a una de ellas registrada como hombre, se podría enfrentar a la cárcel, sanciones y anulación de su unión ${ }^{13}$, porque en Costa Rica la única unión civil válida hasta la fecha es la efectuada entre un hombre y una mujer.

«Guardar las apariencias» es otro punto importante. Muchas personas LGBT a veces no saben bien cómo enfrentar la realidad de lo que son y por temor se resguardan en relaciones heterosexuales que quizás les espanten la soledad, pero nunca les llenarán el vacío que la falta de aceptación les provoca. Como ejemplo de esto se puede ver en el cuento «Bienvenido a tu nueva vida» de Uriel Quesada, relato que en su momento de publicación en el periódico La Nación, causó gran polémica por el lenguaje explícito y erótico con el que representó una situación tan común como lo es un viaje en tren y el sinfín de rostros e historias que se pueden ver pasar por un andén y sus vagones.

[...] Procuro concentrar mi atención en el paisaje. Suspiro y cierro los ojos, intento ver al joven de la flor. Pero en medio de mi ensoñación siento que algo roza mi pierna. No es una sensación desconocida. Sé además que me gusta y que en ocasiones

13 Murillo, A. (2015). Periódico digital español El País. como esa me quedo quieto, relajado, en espera a que la caricia avance. Entreabro los ojos y la pareja sigue besándose, pero el pie del chico, libre del cuartelito del zapato busca meterse bajo mi pantalón ${ }^{14}$.

[...] De repente él sale, se vuelve hacia la chica y le dice alguna cosa. No para de sonreírle. Cierra la puerta y quedamos los dos en el pasillo. Camina hacia mí pero parece que no me ve. Me hago a un lado para dejarlo pasar. Entonces su mano enorme y bella, con el anillo reluciente, se posa sobre mi estómago mientras él sigue avanzando. Hace una levísima presión, pero es suficiente para aclarar el mensaje. Me dejo ir. Unos pasos más allá, la portezuela del baño nos invita ${ }^{15}$.

Entramos a ese mundillo vertical, reducido, destinado al trámite rápido. Tenemos muy poco espacio, pero de todas formas nuestros cuerpos no desean guardar ninguna distancia. El primer beso sabe a perfume de mujer y desesperación. Los siguientes tienen sabor a macho en celo ${ }^{16}$.

En los fragmentos anteriores, se ve a una pareja demostrándose afecto $\mathrm{y}$ en frente de ellos va sentado otro muchacho. La sonrisa cómplice de la chica se puede inferir como conocedora de la situación de su pareja, no solo eso, lo acepta y prácticamente anima a que su hombre prosiga con lo que quiere. ¿Es acaso una máscara protectora de su pareja para resguardar las apariencias? Puede ser, aunque eso no esté explícitamente escrito en las líneas que muestra Quesada con este relato y aun así

14 Obando, A. (2008: 175,176). Autor original Uriel Quesada.

15 Obando, A. (2008: 175,176). Ídem.

16 Obando, A. (2008: 175,176). Ídem. 
es un buen retrato de varios que talvez han recurrido a un buen o buena amiga, capaz de comprender el pavor que les provoca ser etiquetados como «no normales» por un gusto que difiere del tradicional.

Son diversos y amplios los ejemplos de ficción literaria que muestran situaciones que viven las personas LGBT; enseñan que son como todo el resto de seres humanos, que escogen una carrera, escriben un e-mail a una amiga o viajan en tren; sus intimidades tienen la única variante de compartir cuerpo, deseo, afecto y amor con un individuo del mismo sexo.

Al cierre de este trabajo, persiste la interrogante, ¿por qué la sociedad (costarricense en este caso) sigue viendo con ojos de censura a estas personas? Quizás, todavía, falten más años o décadas para llegar a una completa aceptación de la diversidad de los seres humanos. Diversidad en sus colores, culturas, tradiciones, religiones $\mathrm{y}$ orientaciones sexuales, pero humanos todos y cada uno de ellos. No obstante, para llegar a la aceptación hay que hablar, conversar de esto desde temprana edad, en la casa, escuela y colegio; dejar de verlo como tabú, hacer conocer obras literarias cuya temática logre desvendar los ojos 1lenos de tradición discriminatoria y muestren como parte de la cotidianeidad vidas libres de temor a la aceptación de su orientación sexual.

\section{Bibliografía}

Arango de Montis, I. (2008). Sexualidad humana. México: Editorial Manual Moderno. Recuperado de: https: //books.google.es/ books?id=S-r6CAAAQBAJ\&pg $=$ PT $185 \& \mathrm{dq}=$ orientaci $\% \mathrm{C} 3 \%$ B3 $\mathrm{n}+\mathrm{sexual} \& \mathrm{hl}=\mathrm{es} \& \mathrm{sa}=\mathrm{X} \& \mathrm{ved}=0 \mathrm{a}$ hUKEwjQmqi4wJrQAhWEQSYKHT_QDLo4ChDoAQgqMAQ $\# \mathrm{v}=$ onepage \&q=orientaci $\% \mathrm{C} 3 \% \mathrm{~B}$ $3 \mathrm{n} \% 20$ sexual \&f=false

Maroto, A. (2006). Homosexualidad y Trabajo social: herramientas para la reflexión e intervención profesional. Madrid: Consejo General de Colegios Oficiales de Diplomados en Trabajo Social y Asistentes Sociales. España. Recuperado de: https://books.google.es/books?id= 5 w54o3J31iMC\&pg=PA301\&dq= orientaci\%C3\%B3n+sexual $\& \mathrm{hl}=$ es\&sa=X\&ved=0ahUKEwjauvPu sp_QAhUmrVQKHTpnCnkQ6AEI QDAI\#v=onepage \&q=orientaci\%C $3 \%$ B 3n $\% 20$ sexual $\& \mathrm{f}=$ false

Murillo, A. (2015). Un 'error' permite el primer matrimonio gay en Centroamérica. El País. Internacional. España. Recuperado de: http://internacional.elpais.com/ internacional/2015/11/05/actualidad/1446679918_194388.html

Obando, A. (2008). La gruta y el arcoíris: Antología de narrativa gay/lésbica costarricense. San José: Editorial Costa Rica. 
Serrano, J. F. (2006). Otros cuerpos, Otras sexualidades. Bogotá: Instituto Pensar. Pontificia Universidad Javeriana. Recuperado de: https://books.google.co.cr/boo $\mathrm{ks}$ ? id=GJ spj3F5Pi4C\&pg =PA $162 \& \mathrm{dq}=\operatorname{lgbt}+$ definici $\% \mathrm{C} 3 \% \mathrm{~B}$ $3 \mathrm{n} \& \mathrm{hl}=\mathrm{es}-419 \& \mathrm{sa}=\mathrm{X} \& \mathrm{redir}$ $\mathrm{esc}=\mathrm{y} \# \mathrm{v}=$ onepage $\& \mathrm{q}=\operatorname{lgbt} \% 20$ definici\%C3\%B3n\&f=false
Ulloa, D. (2016). En el país de Nunca Jamás: oda a los bares gays. Especial de Revista Dominical. La Nación. Costa Rica. Recuperado de: http://www.nacion.com/ ocio/revista-dominical/pais-Jamas_0_1569243118.html 